\title{
Numerical investigations on pressurized AL-composite vessel response to hypervelocity impacts: Comparison between experimental works and a numerical code
}

\author{
Jérôme Mespoulet ${ }^{\mathrm{a}}$, Fabien Plassard, and Pierre-Louis Hereil \\ THIOT INGENIERIE, Route Nationale, 46130 Puybrun, France
}

\begin{abstract}
Response of pressurized composite-Al vessels to hypervelocity impact of aluminum spheres have been numerically investigated to evaluate the influence of initial pressure on the vulnerability of these vessels. Investigated tanks are carbonfiber overwrapped prestressed Al vessels. Explored internal air pressure ranges from 1 bar to 300 bar and impact velocity are around $4400 \mathrm{~m} / \mathrm{s}$. Data obtained from experiments (Xray radiographies, particle velocity measurement and post-mortem vessels) have been compared to numerical results given from LS-DYNA ALE-Lagrange-SPH full coupling models. Simulations exhibit an under estimation in term of debris cloud evolution and shock wave propagation in pressurized air but main modes of damage/rupture on the vessels given by simulations are coherent with post-mortem recovered vessels from experiments. First results of this numerical work are promising and further simulation investigations with additional experimental data will be done to increase the reliability of the simulation model. The final aim of this crossed work is to numerically explore a wide range of impact conditions (impact angle, projectile weight, impact velocity, initial pressure) that cannot be explore experimentally. Those whole results will define a rule of thumbs for the definition of a vulnerability analytical model for a given pressurized vessel.
\end{abstract}

\section{Introduction}

The context of this paper is the vulnerability of high pressure vessels subjected to high velocity impact of space debris. The increase number of these debris leads to a new regard on these phenomena. From an operational point of view, it is essential to know the limit between the perforation regime and the bursting regime of the vessel in function of the internal pressure and of the projectile impact characteristics (velocity, mass, impact angle ....).

One of the first study on this topic has been performed by NASA in 1963 [1]. Experimental investigations consist in the impact into liquid-filled tanks. The goal was to study the effects of some of the primary variables contributing to bursting or catastrophic fracturing of liquid-filled tanks. A lot of studies have been done by NASA and SwRI on this subject [2-5].

Since 1995, EMI and ESA group has presented a lot of experimental, numerical and analytic works on the vulnerability of high pressure vessels [6-10]. Interesting experimental results are the visualization of debris cloud in high pressure gas and the deceleration of this debris by this gas. Majority of tests have been realized at normal incidence, at velocity around $7 \mathrm{~km} / \mathrm{s}$ with aluminum spheres as projectiles. The impact tests were performed on cylindrical pressure vessels which were unshielded or shielded and made of AL5754, A12219, unalloyed Ti or Ti6Al4V. Most of the cylindrical vessels had a wall thickness of $1 \mathrm{~mm}$, a diameter of $150 \mathrm{~mm}$ and a length of $350 \mathrm{~mm}$.

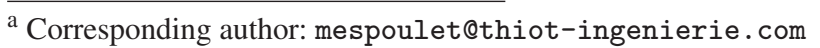

In 2001, a French group (EADS, INSA, CNRS and CNES) has presented some numerical simulation with SPH algorithm on the behavior of high pressure composite tanks under an hypervelocity impact. This numerical study has been used to prepare a following experimental campaign on composite tanks [11,12].

The aim of the work presented in this paper, is to numerically analyze the different regimes of a pressurized composite/metal vessel subjected to an hypervelocity impact. These regimes are the penetration mode, the critical rupture and fragmentation mode, and finally the bursting mode. Driving parameters are impact velocity, debris characteristics and internal pressure level. The effects of internal gas pressure vessel on the state of the vessel will be analyzed in the impact regime of an $8 \mathrm{~mm}$ diameter aluminum sphere at an impact velocity of $4400 \mathrm{~m} / \mathrm{s}$. The first difference with previous published studies is the tested vessel which is an overwrapped carbon fiber on an aluminum liner. The second difference concerns the value of the internal pressure explored, which will be 300 bar, the maximum pressure admitted in this vessel of a volume of 1.1 liter.

The results presented in this paper are based on a more important program supported by CNES and which includes hypervelocity impact experiments performed with some specific measurements in order to have enough data to discriminate the calculations. All these results are compared to numerical simulations in order to evaluate the reliability of such calculations in this specific field of hypervelocity impacts against high pressure vessels.

The stake of numerical simulations of high pressure vessels vulnerability is to be able to extrapolate the 
Table 1. Parameters of the hypervelocity impact tests campaign.

\begin{tabular}{|l|l|l|}
\hline Test n & Projectile velocity (m/s) & Vessel pressure (bar) \\
\hline \#HE0183 & $4334 \pm 60$ & 1 \\
\hline \#HE0184 & $4325 \pm 60$ & 200 \\
\hline \#HE0188 & $4310 \pm 60$ & 300 \\
\hline
\end{tabular}

experimental results at velocities up to $16 \mathrm{~km} / \mathrm{s}$ which are not achievable in laboratory on Earth.

The challenge of theses simulations is to combine several complex effects with a full $3 \mathrm{D}$ model. The aim of this paper is to evaluate the capability of commercial codes to reproduce the different events that occur during experimental tests.

\section{Experimental work}

The hypervelocity impact tests were performed with the HERMES two-stage light gas gun at Thiot Ingenierie Laboratory [13]. Vessels used in this study are used for paintball games. No details on the manufacturing and material characteristics are given by the supplier but the technology of these vessels seems to be closed to the technology of composite tanks that are used in satellites. Part of hypervelocity impact test campaign at $90^{\circ}$ impact angle that have been numerically investigated are presented in Table 1.

Analysis of the data and the results obtained during the four tests of hypervelocity impact on compositeAluminum vessel confirms the results already presented on pressurized vessel: the velocity of the debris cloud generated by the impact is reduced by the effect of the gas. Failure of the pressurized vessel increases with the inflated pressure, but for all the pressure investigated (200-300 bar) failure mode is the same: it leads to 2, 3 or 4 main debris of the aluminum layer of the vessel and a highly damaged state for the composite layers. Failure mode is directly dependent of the composite texture. The impact of the aluminum sphere leads to a hole in the aluminum liner and to the rupture of the composite fibers. The confining stress induced by these overwrapped fibers is then released in this circumferential portion which results in the rupture of the aluminum portion in this same location.

\section{Description of numerical simulations}

\subsection{Context}

Simulations have been done using the nonlinear explicit code LS-DYNA. This study combines several complex problems with strong interferences between them:

- Multi layered vessel made of aluminum liner prestressed by external tension fibers winding composite materials,

- Rupture modes of these materials at hypervelocity impact,

- Initialization of the gas pressure inside the vessel with replication of shock waves generated by impact,

- Complete interactions between projectile/vessel, projectile/gas and vessel/gas.

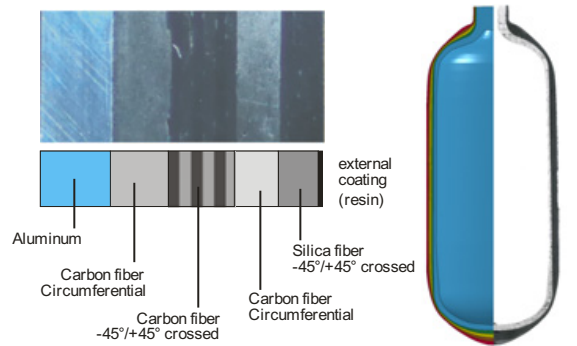

Figure 1. Cut view with description of the Aluminum-Composite vessel (left) and comparison of cut-view with model (right).
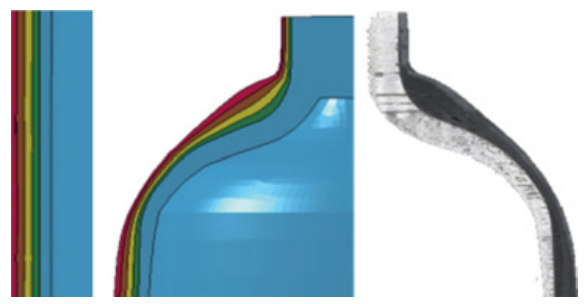

Figure 2. Comparison of vessel end cut-view with model numerical (left) and detail of the model impact area (right). (a)

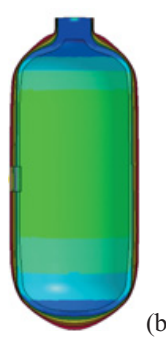

(b)

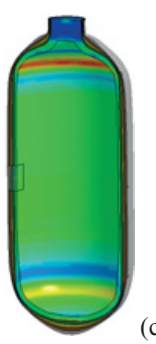

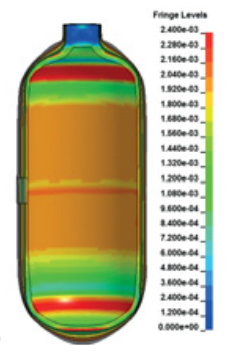

Figure 3. Comparison of initial Von Mises stress (0-240 MPa) in aluminum layer (a) at 0 bar (pres-stress at rest), (b) 200 bar and (c) and 300 bar.

\subsection{Solvers, material laws and interactions}

The vessel is modeled with a lagrangian formulation, using the real thickness variations taken from the half cross section of the vessel (Fig. 1). The aluminum liner thickness varies between 2 and $4 \mathrm{~mm}$. The composite external material is made of 4 plies layered with: carbon fiber circumferential, carbon fiber $-45^{\circ} /+45^{\circ} \mathrm{C}$ rossed, carbon fiber circumferential and silica fiber $-45^{\circ} /$ $+45^{\circ} \mathrm{C}$ rossed. Meshing are done with hexagonal solid elements with 0.5 to $2 \mathrm{~mm}$ segment characteristic length.

Comparison of tested vessel cut-views with model are presented on Fig. 1 (large view) and in details (Fig. 2).

A static nonlinear step is first performed to take into account the prestresses induced by the composite fibers winding. Without this step, calculations show that the vessel is not able to withstand an internal pressure of 300 bar. A mean consistent value of $100 \mathrm{MPa}$ Von Mises stresses is arbitrarely chosen for the aluminum liner as initial compressive stress at rest. A calculation with the desired values of gas internal pressure is then performed according to the initial pressure in the experimental test (Fig. 3).

Air inside and outside the vessel is modeled with an ALE formulation. The vessel is totally immersed in the 


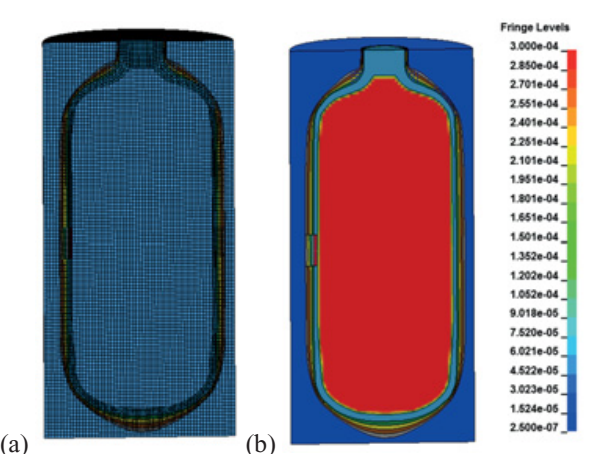

Figure 4. (a) Meshing view of Lagrangian parts immerged in ALE fluid part, (b) Fluid/structure interaction model with 300 bar initial pressure.

ALE mesh. Air inside the vessel is initialized with the same pressure as in experiment (Fig. 4). Air outside the vessel is initialized with a partial vacuum of $250 \mathrm{mbar}$. A perfect gas equation of state is used for Air in ALE grid. The stress/strain state (prestresses + internal pressure) is taken as initial state of explicit impact calculations.

The $8 \mathrm{~mm}$ diameter aluminum sphere is modeled with SPH formulation. The SPH solver was first introduced in the seventies for astrophysics. It is a Lagrangian method with a variable nodal connectivity. It is a gridless method which can handle severe distortions without grid tangling and so does not need the use of erosion algorithms. This technique is then well adapted for hypervelocity phenomena simulations [10-12,14-16].

The aluminum parts are modeled with a classic elastoplastic model with an erosion criterion based on strain. The composite layers are modeled with a composite material model with 9 elastic constitutive parameters and a ChangChang failure model criterion based on the brittle limit for each direction.

The main difficulty is the correct treatment of the interfaces between each formulation:

- Interaction between SPH sphere and vessel modeled with 3D Lagrangian elements is treated with a node/surface interface,

- Interaction between vessel and air is managed with a fluid/structure interface,

- Interaction between the ball and the gas is managed by a recently developed interface between SPH and ALE formulation. At this time, this interaction capability is only available with a beta version of Ls-Dyna program $[16,17]$.

\section{Results and comparison with experiments}

\section{1. \#HE0183}

The first test simulated is \#HE0183 which is characterized by an internal pressure of 1 bar inside the composite vessel. For this test, the shock wave generated in the gas is not decisive and so calculation is performed without ALE grid.

The shape of aluminum rupture (Fig. 5) does not reproduce exactly the experiment but it can be explained

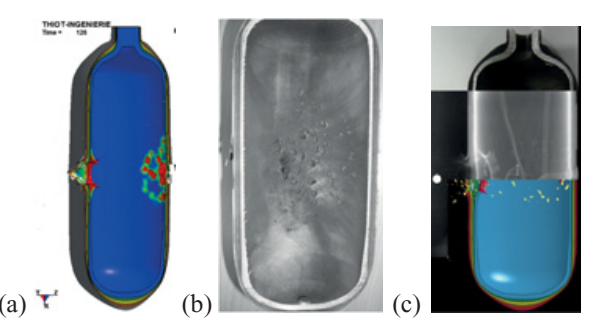

Figure 5. HE\#183: Illustration of damaged zones at the inside rear face obtained (a) in simulation, (b) in experiment.; (c) Comparison between calculation and Xray radiography $41 \mu \mathrm{s}$ after impact.

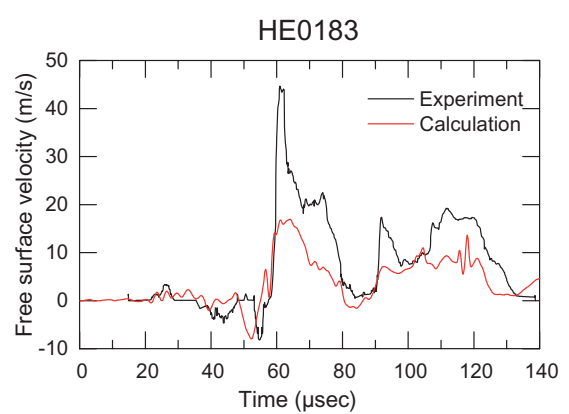

Figure 6. HE\#183: Experiment and calculation comparison of the free surface velocity.

by the fact that the mesh is too coarse to simulate this phenomenon and the rupture model would need to be improved. The direction and the shape of composite damages are consistent with the experimental test. Fragments do not pass through the vessel skin but damage on external side is consistent with experiment. The last picture on this figure shows a comparison with the Xray radiography taken $41 \mu$ s after impact. We can see that this time corresponds to the arrival time of the debris cloud on the opposite side of the vessel.

Figure 6 shows a comparison of the free surface particle velocity. From a chronometry point of view, calculation is consistent with experiment.

\section{2. \#HE0184}

Test HE0184 has been performed with an internal air pressure of 200 bar. In this configuration, taking into account fluid is essential to obtain the global behavior. Indeed, pressure build up acting on inside face increases the initial stress in the aluminum liner and amplifies tension in composite external skin.

Figure 7 shows the evolution of shock waves in the pressurized air and the motion of the SPH nodes after impact. The fragmentation of the projectile is not well reproduced. Some numerical tests have shown that the interface between SPH and ALE is so high that behavior of SPH part is misrepresented. No more expansion of SPH nodes occurs when nodes interact with ALE fluid material. The projectile is almost stopped by the gas and fragment impacts the opposite of the aluminum liner at low velocity $(<200 \mathrm{~m} / \mathrm{s})$. This phenomenon is consistent with the experimental observation. 


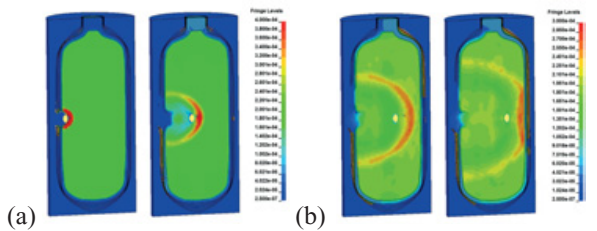

Figure 7. HE\#184: Evolution of shock wave pressure in pressurized air (a) 30 and $85 \mu \mathrm{s}(0-400$ bar) and (b) 153 and $208 \mu$ s after impact (0-300 bar).

(a)

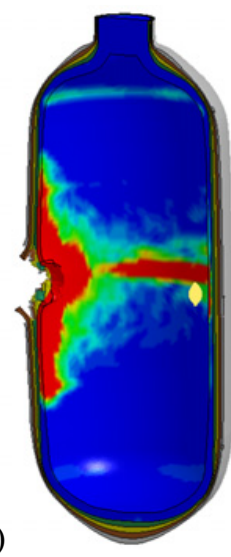

(b)

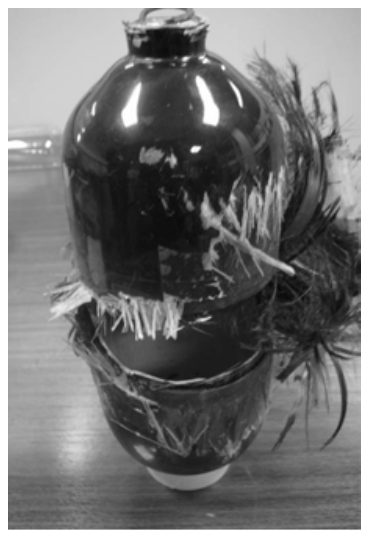

Figure 8. HE\#184: (a) Inside view of simulation damaged zones in Al liner, (b) Postmortem reservoir after \#HE0184.

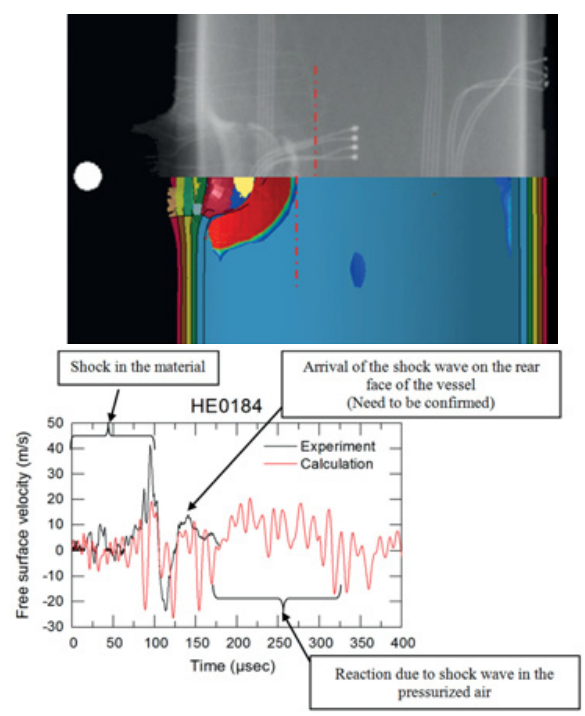

Figure 9. HE\#184: Comparison between calculation and Xray radiography $41 \mu \mathrm{s}$ after impact (top), Test and calculation comparison of the free surface velocity (bottom).

The shock wave in the gas reaches the opposite side $140 \mu$ s after impact. Calculations indicate that the vessel rupture is caused by initial pressure that acts on internal side of the vessel and opens it. This rupture is not total which is consistent with the real case but the damaged zone is not reproduced (Fig. 8).

Figure 9 shows the comparison with the Xray radiography. Shock wave in the vessel is reproduced, but its propagation velocity is slower than in the numerical

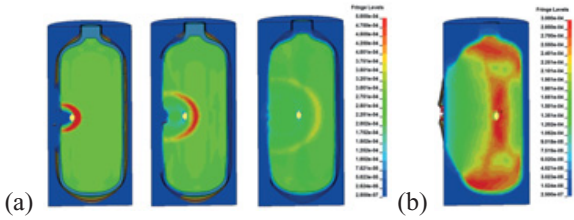

Figure 10. HE\#188: Evolution of shock wave pressure in pressurized air (a) 48, 89 and $144 \mu \mathrm{s}(0-500$ bar) and (b) $300 \mu \mathrm{s}$ after impact ( $0-48$ bar).

model (shock front is symbolized by red axis lines). Hypothesis of this discrepancy have been listed below:

- aluminum liner deformation is less than in simulation, so a smaller amount of gas is initially pushed by the liner deformation,

- an ideal gas is probably not sufficient to reproduce the real physic, especially shock velocity in this high pressure state,

- Interaction between the ball and the gas is managed by a meshing size should be reduced,

- strain rate dependent model should be used.

Figure 9 shows also the free surface particle velocity. Initial oscillations can be explained by penalty algorithm implementation for dynamic treatment of the fluid/structure. Nevertheless, the first peak corresponding to shock waves propagation in vessel material at $95 \mu \mathrm{s}$ and the effect of the shock wave in the air with duration of around $125 \mu$ s can be distinguished.

\section{3. \#HE0188}

Test HE0188 is performed with an internal air pressure of 300 bar. Figure 7 shows the evolution of shock waves in the pressurized air and the motion of the SPH nodes after impact. Once again, the fragmentation of the projectile is not well reproduced: no expansion of SPH nodes after ALE interaction. The projectile is once again stopped by the gas as in experiment. The shock wave in the gas reaches the opposite side at $160 \mu \mathrm{s}$. We can see that rupture of the vessel is caused by initial pressure. The pressure on the ruptured zone is responsible for the opening of the vessel. Damage on the vessel occurs before reflection of the shock wave on the opposite side of the vessel.

Figure 11 shows the rupture of the vessel with the same large parts as in the experiment. Simulation give two caps and the fractured body but sizes are not exactly the same than the test. Once again a finer mesh and a better knowledge of the individual behavior of material that compose the vessel (especially composites) needs to be investigated.

Figure 12 shows the comparison with the Xray radiographies for two different times. We can see that the shock wave in the vessel is reproduced. Once again, the shock wave propagation velocity is slower in the numerical model due probably to hypothesis explained in the previous paragraph

Figure 13 shows the free surface particle velocity at the rear face of the vessel. The oscillations at the beginning can be explained by the dynamic treatment of the fluid/structure with implementation of the penalty 

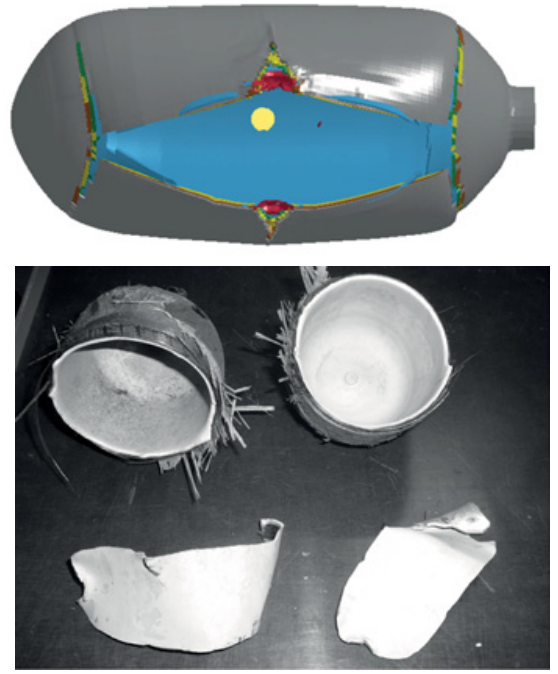

Figure 11. HE\#188: Inside view of damaged zones in Al liner, (top), Postmortem reservoir after \#HE0188 (bottom).

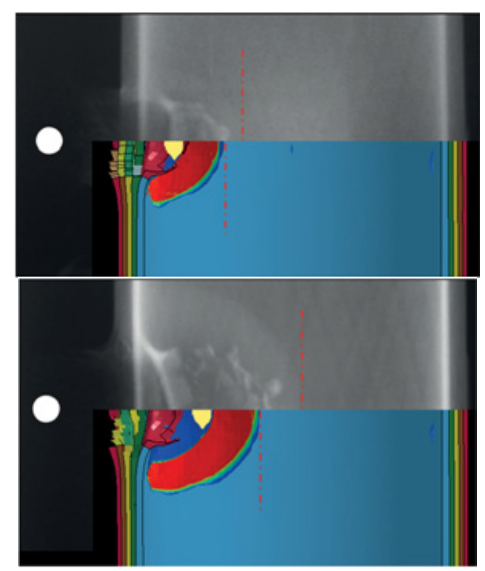

Figure 12. HE\#184: Comparison between calculation and Xray radiography $13 \mu$ s after impact (top), $28 \mu$ s after impact (bottom).

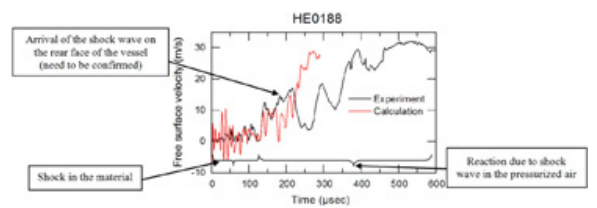

Figure 13. HE\#188: Experiment and calculation comparison of the free surface velocity).

algorithm. Nevertheless we are able to distinguish some peaks corresponding to the propagation of the shock wave in the vessel material around $100 \mu \mathrm{s}$. Then the velocity of the rear side of the exploded vessel increases.

\section{Analysis}

Figure 15 presents the evolution of the projectile velocity versus time for the three studied cases: HE00183 not pressurized, HE0184 pressurized at 200 bar and HE00188 pressurized at 300 bar. This graph shows the effect of the high pressure gas on the velocity decrease of the projectile.

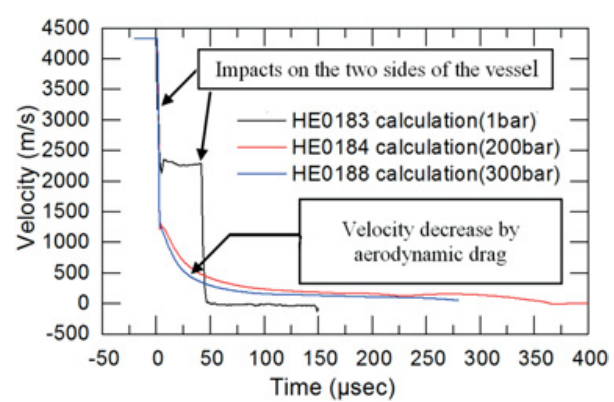

Figure 14. Evolution of the projectile versus time for the three simulations \#HE0183, \#HE0184 and \#HE0188.

At the impact on the first side, the non-pressurized vessel decreases the projectile velocity at $2250 \mathrm{~m} / \mathrm{s}$, while pressurized vessels decrease the projectile at $1250 \mathrm{~m} / \mathrm{s}$. The fragments are stopped due to aerodynamic drag in the pressurized gas.

\section{Conclusions and future works}

The responses of gas-filled pressure composite-aluminum vessels subjected to hypervelocity impact are numerically analyzed from selected experimental results. It has been found during experimental phase that failure of the pressurized vessel increases with the inflated pressure and that failure mode depends directly on the stacking sequence of composite.

The numerical study developed in this paper has shown that a commercial code as Ls-Dyna has tools to reproduce global phenomenon from experimental setup. A crossed study between experiments and calculations allows us to understand the storyline of the hypervelocity impacts on pressurized composite vessels. The main difficulty is the management of interactions between SPH formulations, Lagrangian finite elements and ALE element for gas. However, an important work is still needed to improve the consistency and the robustness of the code. Especially for the new SPH/ALE interaction which shows limits in term of physics. Materials knowledge of the studied vessel has also to be improved in order to fill the code with right parameters in material models.

The authors thank CNES and Thiot Ingenierie for funding. In addition, the authors appreciate the support provided by Thiot-Ingenierie Facility and personnel including Valérie Berger, Ghislain Darnis and Fabrice Vigié.

\section{References}

[1] F.S. Stepka, R.P. Dengler, C.R. Morse, Preliminary Investigation of Catastrophic Fracture of LiquidFilled Tanks Impacted by High-Velocity Particles, NASA Technical Note D-1537, LeRC, OH (1963)

[2] J.P. Whitney and P.D. White, Designing hypervelocity impact testing for thin-walled pressure vessels, Proceeding of the $43^{r d}$ ARA meeting, Columbus, $\mathrm{OH}$ (1992) 
[3] J.P. Whitney, Hypervelocity impact tests of shielded and unshielded pressure vessels, NASA Johnson Space center,Report JSC-32294 (1993)

[4] E. Christiansen, J.H. Kerr and J.P. Whitney, Debris cloud ablation in gas-filled pressure vessels, Int. J. of Impact Engng 20, 7 (1997)

[5] S.A. Mullin, H. Couque, B.G. Cous-Palais, B.G. Grosch and J.D. Walker, Bursting of shielded pressure vessels subject to HVI, Int. J. of Impact Engng 20, 7 (1997)

[6] M. Lambert and E. Schneider, Hypervelocity impacts on gas filled pressure vessels, Int. J. of Impact Engng 20, 7 (1997)

[7] F.E. Schäfer, E. Schneider and M. Lambert, An experimental study to investigate HVI on pressure vessels, Second European Conference on Space Debris, Darmstadt, Germany (1997)

[8] F.E. Schäfer, E. Schneider and M. Lambert, Impact fragment cloud propagating in a pressure vessel, Act. Astr. 39, 1 (1997)

[9] I.Y. Telitchev, F.E. Schäfer and E. Schneider, Hypervelocity impacts on gas filled pressure vessels, Int. J. of Impact Engng 23, 7 (1999)

[10] M. Palmieri, F.E. Schäfer, S. Hiermaier and M. Lambert, Numerical simulation of non-perforating impacts on shielded gas-filled pressure vessels, Int. J. of Impact Engng 26, 7 (2001)
[11] C. Maveyraud, J.P. Vila, D. Sornette, C. Le Floch, J.M. Dupilier and R. Salomé, Numerical modelling of the behavior of high pressure vessel tank under an hypervelocity impact, Proceeding of the $3^{\text {rd }}$ Int. Conf. on Spacecraft Propulsion, ESA (2000)

[12] R. Salomé V. Albouys, C. Le Floch, D. Sornette and J.P. Vila, High pressure composite tank behavior under an hypervelocity impact, ESA (2001)

[13] P. Thiot, P.L. Héreil, J. Mespoulet, G. Darnis, V. Gasquet, W. Chan and K. Taylor, Technical description and capabilities of THIOT INGENIERIE's TwoStage Light-Gas-Gun, Proceeding of the $60^{\text {th }}$ ARA meeting, Baltimore (2009)

[14] L.D. Libersky and A.G. Petscheck, High Strain Lagragian Hydrodynamics, J. of Comp. Phys. 109 (1993)

[15] F. Plassard, J. Mespoulet and P.L. Héreil, Hypervelocity impact of aluminium sphere against aluminium plate: experiment and LSDYNA correlation, Proceeding of the $10^{\text {th }}$ Inter. LS-Dyna Users conf., Starsbourg (2011)

[16] H. Chen and J. Wang, LS-Dyna ALE nodal coupling, Proceeding of the $12^{\text {th }}$ Inter. LS-Dyna Users conf. (2012)

[17] Recent developments in LSs-Dyna, LS-Dyna User forum (2013) 